\title{
ORTHOGONAL PROPERTIES OF INDEPENDENT FUNCTIONS
}

\author{
BY \\ SAMUEL KARLIN
}

This paper investigates the orthogonal structure of independent systems of functions. The tendency in dealing with independent functions has been largely to establish results relating to the law of large numbers and central limit theorems (see [1])(1). The treatment here is concerned with an analytical study of the orthogonal properties of independent functions and does not consider probability interpretations.

Two functions $f_{1}(t)$ and $f_{2}(t)$ are said to be independent over the interval $(0,1)$, if for any $\alpha_{i}, \beta_{i}(i=1,2)$ we have

$$
\left|\underset{t}{E}\left(\alpha_{1}<f_{1}(t) \leqq \beta_{1}, \alpha_{2}<f_{2}(t) \leqq \beta_{2}\right)\right|
$$

$$
=\left|\underset{t}{E}\left(\alpha_{1}<f_{1}(t) \leqq \beta_{1}\right)\right|\left|\underset{t}{E}\left(\alpha_{2}<f_{2}(t) \leqq \beta_{2}\right)\right|
$$

where $\mid$ denotes the measure of the set. The extension to several functions is done in a natural manner [4, pp. 61-63].

The classical example of such a system of functions is the well known system of Rademacher. This system can be defined as follows:

$$
\begin{array}{ll}
x_{n}(t)=\operatorname{sign} \sin \left(2^{n+1} \pi t\right) & \text { for } 0 \leqq t \leqq 1, \\
x_{0}(t)=1 . &
\end{array}
$$

Other important systems have been introduced by Steinhaus [2, pp. 2327], and $\mathrm{Kac}[3, \mathrm{p} .64]$.

Some immediate consequences of the definition are

(a) If $x_{1}(t), \cdots, x_{n}(t)$, are independent, then

$$
\int_{0}^{1} \prod_{1}^{n} x_{i}(t) d t=\prod_{1}^{n} \int_{0}^{1} x_{i}(t) d t .
$$

If we assume the mean value of each $x_{n}(t)$ equal to zero, then the orthogonality of $x_{n}(t)$ results from (2). Another result is that if $x_{1}+\cdots+x_{n}$ belong to $L^{p}$, then each $x_{n}(t)$ belongs to $L^{p}$ for $p \geqq 1$. It is to be remarked now that $x_{n}(t)$ considered as an orthogonal system is incomplete, that is, there exist nonzero functions (for example, $g(t)=x_{1}(t) x_{2}(t)$ ) which are orthogonal to all $x_{n}(t)$. Consequently, no uniqueness of expansions can be expected.

Presented to the Society, February 26, 1949; received by the editors December 13, 1947.

(1) Numbers in brackets refer to the references cited at the end of the paper. 
Independent functions are important for their connection with independent random variables, and on the other hand, for any sequence of measurable functions $f_{n}(t)$, there exists a sequence of independent functions $x_{n}(t)$ which are equimeasurable to $f_{n}(t)$ respectively [4, pp. 62-63].

This paper is divided into three main sections dealing with convergence, summability, and lacunarity respectively. In $\$ 1$ convergence in $L^{p}$ and ordinary pointwise convergence are discussed. $\$ 2$ treats relationships of summability to convergence. Finally, in $\$ 3$ the connection of lacunarity and independence systems with lacunary orthogonal systems is considered.

Whenever an integral is written we presuppose sufficient assumptions to insure the existence of the integral. The assumption of existence of the integral will always be valid if it is assumed that $\left|x_{m}(t)\right| \leqq M_{n}$, that is, that $x_{m}(t)$ need not be uniformly bounded. Less restrictive conditions can usually be imposed.

The domain of definition of $x_{m}(t)$ is $(0,1)$. The conjugate exponent $p^{\prime}$ to $p$ will be the number such that $1 / p+1 / p^{\prime}=1$.

1. Convergence. In this section we deal with convergence properties of independent series. The first part of this section will be devoted to studying modes of convergence of partial sums and their interrelationships.

Let $x_{n}(t)$ be an independent system of functions each possessing a mean value zero, $\int_{0}^{1} x_{n}(t) d t=0$. This assumption implies that the $x_{n}(t)$ can be considered as an orthogonal system of functions. We do not at first assume that the system is normalized.

An essential tool which will be used frequently throughout the paper is the following lemma established by Marcinkiewicz and Zygmund [5, pp. 109-115].

LEMMA 1. If $\int_{0}^{1} x_{n}(t) d t=0$, then for all $m$

$$
\begin{array}{ll}
\int_{0}^{1} \max _{1 \leqq n \leqq m}\left|s_{n}(t)\right|^{p} d t \leqq K \int_{0}^{1}\left|s_{m}(t)\right|^{p} d t & \text { for } 1 \leqq p \leqq \infty, \\
A_{p}^{\prime} \int_{0}^{1}\left(\sum_{1}^{m} x_{n}^{2}\right)^{p / 2} \leqq \int_{0}^{1}\left|s_{m}(t)\right|^{p} d t \leqq A_{p} \int_{0}^{1}\left(\sum x_{n}^{2}\right)^{p / 2}
\end{array}
$$

for $1 \leqq p<\infty$, where $s_{m}(t)=\sum_{1}^{m} x_{n}(t)$ and $A_{p}, A_{p}^{\prime}$ are constants depending only on $p$.

We first establish a lemma which is fundamental in all that follows.

Lemma 2. A necessary and sufficient condition that $\sum_{1}^{m} a_{l} x_{l}(t)=s_{m}(t)$ converge to a function belonging to $L^{p}(1 \leqq p<\infty)$ is that

$$
\int_{0}^{1}\left|s_{m}(t)\right|^{p} d t \leqq \gamma \quad \text { for } m=1,2, \cdots \text {. }
$$


Proof. Necessity. In virtue of Lemma 1 and the fact that $s(t)=\sum_{1}^{\infty} a_{n} x_{n}(t)$ is in $L^{p}$, we have

$$
\int_{0}^{1}\left|s_{n}(t)\right|^{p} d t \leqq \int_{0}^{1} \max _{n}\left|s_{n}(t)\right|^{p} d t \leqq K \int_{0}^{1}|s(t)|^{p} d t \leqq \gamma .
$$

Sufficiency. Since $\int_{0}^{1}\left|s_{n}(t)\right|^{p} d t \leqq \gamma$ we have by Lemma 1 that

$$
\int_{0}^{1}\left|\sum_{1}^{n} a_{m}^{2} x_{m}^{2}(t)\right|^{p / 2} d t \leqq \frac{\gamma}{A_{p}^{\prime}} .
$$

Hence, $\sum_{1}^{m} a_{n}^{2} x_{n}^{2}(t)$ converges a.e. to a $p / 2$ integrable function. Since

$$
G_{m}(t)=\sum_{m}^{\infty} a_{l}^{2} x_{l}^{2}(t) \geqq G_{m+1}(t),
$$

we obtain in employing Lemma 1 and Lebesgue's convergence theorem that

$$
\int_{0}^{1}\left|s_{m}(t)-s_{n}(t)\right|^{p} d t \leqq A_{p} \int_{0}^{1}\left(\sum_{m}^{\infty} a_{l}^{2} x_{l}^{2}(t)\right)^{p / 2} d t \leqq \epsilon
$$

provided $n>m \geqq N_{0}(\epsilon)$. Again, for any $n$ and $m$

$$
\int_{0}^{1} \max _{m \leqq t \leqq n}\left|s_{t}(x)-s_{m}(x)\right|^{p} d x \leqq A_{p} \int_{0}^{1}\left|s_{n}(x)-s_{m}(x)\right|^{p} d x \leqq K .
$$

Therefore, if $F_{m n}=\max _{m \leqq t \leqq n}\left|s_{t}(x)-s_{m}(x)\right|^{p}$ and since $F_{m, n} \leqq F_{m, n+1} \leqq \cdots$, it follows from (3) that $\lim _{n \rightarrow \infty} F_{m, n}=F_{m}$ exists almost everywhere. Moreover, $F_{n} \geqq F_{n+1} \geqq \cdots \geqq 0$ which gives on account of $(2 \mathrm{a})$ that $\lim _{n} F_{n}=0$ a.e. The conclusion of the proof of the theorem is now immediate.

The theorem is also valid for $p=\infty$. It can be shown that the lemma does not remain true for $p<1$.

We present now several applications of this lemma. For completeness, several definitions will now be given.

A sequence of functions $f_{n}(t)$ converges weakly in $L^{p}(1 \leqq p<\infty)$ if for every function $g(t)$ in $L^{p^{\prime}}(M)$, we have that

$$
\lim _{n \rightarrow \infty} \int_{0}^{1} f_{n}(t) g(t) d t
$$

converges.

A sequence of functions $f_{n}(t)$ converges strongly in $L^{p}(1 \leqq p<\infty)$ if

$$
\lim _{m, n \rightarrow \infty} \int_{0}^{1}\left|f_{n}(t)-f_{m}(t)\right|^{p} d t=0 .
$$

A sequence of functions $f_{n}(t)$ converges in the ordinary sense if $f_{n}(t)$ converges pointwise for almost every $t$. 
Finally, a sequence of functions $f_{n}(t)$ converges asymptotically to a function $f_{0}(t)$ if for any $\epsilon>0$

$$
\lim _{n \rightarrow \infty}\left|\underset{t}{E}\left[\left|f_{n}(t)-f_{0}(t)\right| \geqq \epsilon_{0}\right]\right|=0,
$$

where $\mid$ denotes the measure of the set.

A further lemma which is needed will now be given.

LEMMA 2a. If $s_{n_{k}}(t)$ converges a.e. to a function $f(t)$ integrable $L^{p}$, then $s_{n}(t)$ converges a.e. to $f(t)$.

REMARK. The subsequence $n_{k}$ is arbitrary.

Proof. The set of functions

$$
\begin{aligned}
& X_{n_{1}}(t)=a_{1} x_{1}(t)+\cdots+a_{n_{1}} x_{n_{1}}(t), \\
& X_{n_{2}}(t)=a_{n_{1}+1} x_{n_{1}+1}(t)+\cdots+a_{n_{2}} x_{n_{2}}(t),
\end{aligned}
$$

form an independent system, each having a mean value zero. Moreover, since

$$
\left|s_{n_{k}}\right| \leqq \max _{k}\left|s_{n_{k}}\right|
$$

Lemma 1 implies in virtue of the hypothesis and (3a) that

$$
\int_{0}^{1}\left|s_{n_{k}}(t)\right|^{p} d t \leqq \int_{0}^{1} \max _{k}\left|s_{n_{k}}(t)\right|^{p} d t \leqq K \int_{0}^{1}|f(t)|^{p} d t \leqq \gamma .
$$

Also, for $n_{k}<n<n_{k+1}$

$$
\begin{aligned}
\int_{0}^{1}\left|s_{n}(t)\right|^{p} d t & \leqq A_{p} \int_{0}^{1}\left|\sum_{1}^{n} a_{k}^{2} x_{k}^{2}(t)\right|^{p / 2} d t \leqq A_{p} A_{p}^{\prime} \int_{0}^{1}\left(\sum_{1}^{n_{k}+1} a_{n}^{2} x_{n}^{2}(t)\right)^{p / 2} d t \\
& \leqq C_{p} \int_{0}^{1}\left|s_{n_{k+1}}(t)\right|^{p / 2} d t \leqq \gamma^{\prime}
\end{aligned}
$$

This implies in virtue of Lemma 2 that $s_{n}$ converges to $f(t)$ almost everywhere.

THEOREM 1. For any $1 \leqq p<\infty$ the following equivalent statements are valid for $\left\{s_{m}(t)\right\}$ :

(a) Weak convergence in $L^{p}$;

(b) Ordinary convergence to a function belonging to $L^{p}$;

(c) Strong convergence in $L^{p}$;

(d) Asymptotic convergence to a function in $L^{p}$.

Proof. (a) $\rightarrow(\mathrm{b})$. Since weak convergence of $s_{m}(t)$ in $L^{p}$ implies

$$
\int_{0}^{1}\left|s_{m}(t)\right|^{p} d t \leqq \gamma
$$


for an absolute constant $\boldsymbol{\gamma}$ independent of $m$, an application of Lemma 2 gives the result.

(b) $\rightarrow$ (c). This is a consequence of the proof of Lemma 2.

(c) $\rightarrow$ (a), (c) $\rightarrow$ (d). These are well known.

(d) $\rightarrow$ (b). The hypothesis of (iv) yields the existence of a subsequence $s_{n_{k}} \rightarrow f(t)$, where $f(t) \in L^{p}$. An application of Lemma 2 a completes the proof. Q.E.D.

It is to be remarked that in a certain sense the hypothesis of Theorem 1 are best possible. For it is well known that there exists an independent series $\sum_{1}^{\infty} x_{n}(t)$ which converges almost everywhere but which does not converge in norm of $L^{2}$. We construct such an example. Consider the set of functions $g_{n}(t)$ defined on the interval of $(0,1)$ as follows:

$$
g_{n}(t)=\left\{\begin{array}{cr}
a_{n}, & 0 \leqq t \leqq p_{n} / 2, \\
-a_{n}, & p_{n} / 2 \leqq t \leqq p_{n}, \\
0, & p_{n} \leqq t \leqq 1,
\end{array}\right.
$$

where $a_{n}$ shall denote a sequence of real numbers increasing sufficiently rapidly. There exists a sequence of independent functions $x_{n}(t)$ equimeasurable with $g_{n}(t)$ respectively $[4, \mathrm{p} .62]$. Thus each $x_{n}(t)$ has a mean value zero. If we consider the set of $t$ where

$$
\underset{n}{E}=\underset{t}{E}\left(x_{n}(t)=0, x_{n+1}(t)=0, \cdots\right),
$$

then clearly

$$
\underset{n}{E} \leqq \underset{n+1}{E} \leqq \underset{n+2}{E} \leqq \cdots
$$

It follows from the hypothesis on $a_{n}$ that a point of convergence of the series $\sum x_{n}(t)$ necessarily requires that the value $t$ lie in the set $E_{n}$ from some $m$ on.

On account of the independence of $x_{n}(t)$ and the definition of $g_{n}(t)$, we obtain

$$
\text { measure }\left[\prod_{m}^{\infty} E_{n}\right]=\prod_{m}^{\infty} \text { measure } m\left(E_{n}\right)=\prod_{m}^{\infty}\left(1-p_{n}\right) \rightarrow 1
$$

provided that $\sum p_{n}<\infty$. If we evaluate

$$
\int_{0}^{1}\left|\sum_{1}^{m} x_{n}(t)\right|^{2} d t=\sum_{1}^{m} a_{n}^{2} p_{n}
$$

then on choosing $\sum a_{n}^{2} p_{n}=\infty, \sum p_{n}<\infty$ we find that $\sum x_{n}(t)$ converges almost everywhere, but does not converge in the norm of $L^{2}$. In view of Theorem 1 , it is clear that $f(t)=\sum_{1}^{\infty} x_{n}(t)$ cannot belong to any $L^{p}$ class for $p \geqq 1$. 
As an immediate consequence of Theorem 1 and Lemma 2a, we have the following corollary.

COROLlARY 1. Weak, strong or ordinary convergence to a function in $L^{p}(1 \leqq p<\infty)$ of any subsequence $s_{n_{k}}$ implies the same conclusion for the entire sequence.

This statement asserts that if $s_{n}(t)$ does not converge almost everywhere to a function in $L^{p}$, then no subsequence can converge to a function in $L^{p}$.

We apply Lemma 2 in another direction. It is well known [6] that for $p>1$, a necessary and sufficient condition for weak convergence in $L^{p}(p>1)$ of a sequence of functions $\sum_{1}^{m} a_{l} x_{l}(t)=s_{m}(t)$ is that for every $u$, we have

$$
\begin{aligned}
& \int_{0}^{u} s_{n}(t) d t \rightarrow \int_{0}^{u} s(t) \quad \text { where } s(t) \in L^{p}, \\
& \int_{0}^{1}\left|s_{n}(t)\right|^{p} d t \leqq \gamma .
\end{aligned}
$$

If we consider $x_{n}(\theta)=r_{n}(\theta)$ where $r_{n}(\theta)$ are the Rademacher functions, then if

$$
\int_{0}^{u} \sum_{1}^{m} a_{n} r_{n}(\theta) d \theta \rightarrow \int_{0}^{u} f(t) d t
$$

for every $u$ where $f(t)$ is in $L^{p}(p \geqq 1)$, then $\sum_{1}^{m} a_{n} r_{n}(\theta)$ converges almost everywhere to $f(t)$. In virtue of Lemma 2 , this implies

$$
\int_{0}^{1}\left|\sum_{1}^{m} a_{n} r_{n}(\theta)\right|^{p} d \theta \leqq \gamma .
$$

Hence, condition (4) above is necessary and sufficient in the case of the Rademacher functions for weak convergence in $L^{p}$. In the case of a general independent series, we can assert statements of the following form:

If

$$
\begin{gathered}
\int_{0}^{1} \mid s_{n}(t \mid) d t \leqq \gamma, \\
\int_{0}^{u} s_{n}(t) d t \rightarrow \int_{0}^{u} s(t) d t
\end{gathered}
$$

where $s(t)$ is in $L^{p}(p \geqq 1)$, then $s_{n}(t)$ converges weakly to $s(t)$ in $L^{p}$. For, indeed, (6) implies in virtue of Theorem 1 that for every $u$

$$
\int_{0}^{u} s_{n}(t) d t \rightarrow \int_{0}^{u} f(t) d t \text { and } s_{n}(t) \rightarrow f(t)
$$


a.e., where $f(t)$ is in $L$. This together with (7) yields that $f(t)=s(t)$ almost everywhere. Finally, Lemma 2 gives the result.

We proceed now to discuss other questions related to convergence of

$$
s_{n}(t)=\sum_{1}^{n} a_{l} x_{l}(t)
$$

We establish first an important lemma.

LEMмA 3. If $\left|\lambda_{n}\right| \leqq M$, then for $m=1,2, \cdots$,

$$
\int_{0}^{1}\left|\sum_{1}^{m} \lambda_{n} a_{n} x_{n}(t)\right|^{p} d t \leqq k(p, M) \int_{0}^{1}\left|\sum_{1}^{m} a_{n} x_{n}(t)\right|^{p} d t \quad \text { for } 1 \leqq p<\infty .
$$

Proof. A double application of Lemma 1 gives

$$
\begin{aligned}
\int_{0}^{1}\left|\sum_{1}^{m} \lambda_{n} a_{n} x_{n}(t)\right|^{p} d t & \leqq A_{p} \int_{0}^{1}\left|\sum_{1}^{m} a_{n}^{2} \lambda_{n}^{2} x_{n}^{2}(t)\right|^{p / 2} d t \\
& \leqq M^{p} A_{p}^{2} \int_{0}^{1}\left|\sum_{1}^{m} a_{n}^{2} x_{n}^{2}(t)\right|^{p / 2} d t \\
& \leqq M A_{p} A_{p}^{\prime} \int_{0}^{1}\left|\sum_{1}^{m} a_{n} x_{n}(t)\right|^{p} d t
\end{aligned}
$$

THEOREM 2. If $s_{n}(t)$ converges to $f(t)$ a.e., where $f(t) \in L^{p}(1 \geqq p<\infty)$, then

$$
\int f(t) g(t) d t=\sum_{1}^{\infty} a_{n} b_{n}
$$

the sum converges absolutely with $a_{n}$ and $b_{n}$ the Fourier coefficients of $f(t)$ and $g(t)$ respectively with respect to $x_{n}(t)\left[g(t)\right.$ is any function in $\left.L^{p^{\prime}}\right]$.

Proof. The hypothesis implies in virtue of Lemma 1 that $\int_{0}^{1}\left|s_{m}(t)\right|{ }^{r} d t \leqq \gamma$. Applying Lemma 3, we have

$$
\int_{0}^{1}\left|\sum_{1}^{m} \lambda_{n} a_{n} x_{n}(t)\right|^{p} d t \leqq \gamma^{\prime}
$$

This implies in virtue of Theorem 1 that for any $g(t)$ in $L^{p^{\prime}}$

$$
\sum \lambda_{n} a_{n} b_{n}<\infty
$$

for every bounded sequence $\left|\lambda_{n}\right| \leqq 1$. In particular, putting $\lambda_{n}=\operatorname{sign} a_{n} b_{n}$ shows that $\sum\left|a_{n} b_{n}\right|<\infty$. Moreover, clearly

$$
\int f g=\sum a_{n} b_{n}
$$

THEOREM 3. If $s_{n}(t) \rightarrow f \in L^{p}$ almost everywhere for $p \geqq 1$, then $s_{n}(t)$ 
$=\sum_{1}^{M} a_{l} x_{l}(t)$ converges unconditionally (by every rearrangement of its terms $\left.a_{l} x_{l}(t)\right)$ to $f(t)$.

Proof. In virtue of Lemma 2 and Lemma 3, we obtain for any $\left|\lambda_{n}\right| \leqq 1$

$$
\int_{0}^{1}\left|\sum_{1}^{m} \lambda_{n} a_{n} x_{n}(t)\right|^{p} d t \leqq \gamma
$$

Choosing $\lambda_{n}$ to be 0 or 1 , we obtain

$$
\int_{0}^{1}\left|\sum_{1}^{m} a_{n_{i}} x_{n_{i}}(t)\right|^{p} d t \leqq \gamma
$$

for every subseries. This in virtue of a well known result [6, chap. 1] gives the unconditional strong convergence in $L^{p}$. Applying Theorem 1 yields the ordinary unconditional convergence. To show that every rearrangement converges to the same function, we have by Lemma 1 for $M$ sufficiently large that for any $N>M$

$$
\int_{0}^{1}\left|\sum_{1}^{N} a_{n(q)} x_{n(q)}(t)-\sum_{1}^{N} a_{n} x_{n}(t)\right|^{p} d t \leqq A_{p}^{\prime} \int_{0}^{1}\left|\sum_{k=L(M)}^{\infty} a_{n}^{2} x_{n}^{2}\right|^{p / 2} d t \leqq \epsilon .
$$

Moreover, since

$$
\int_{0}^{1}\left|f(t)-\sum_{1}^{N} a_{n} x_{n}(t)\right|^{p} d t \rightarrow 0, \quad \int\left|g(t)-\sum_{1}^{N} a_{n(q)} x_{n(q)}\right|^{p} d t \rightarrow 0,
$$

we infer that $f(t)=g(t)$ almost everywhere.

Theorem 4. If $\left|\sum_{1}^{m} a_{n} x_{n}(t)\right| \leqq K$ a.e., then $\sum_{1}^{\infty}\left|a_{n} x_{n}(t)\right|<\infty$ a.e.

Proof. We first deal with the case of Rademacher functions $r_{n}(\theta)$. Let us suppose that $\left|\sum_{1}^{m} a_{n} r_{n}(\theta)\right| \leqq K$ a.e. We introduce the positive kernel $\coprod_{1}^{m}\left(1+r_{k}(\theta)\right)$. For any $\left|\lambda_{k}\right| \leqq 1$, on account of the independence of $r_{n}(\theta)$,

$$
\begin{aligned}
\sum_{1}^{m} a_{n} \lambda_{n} & =\int_{0}^{1} \sum_{1}^{m} a_{n} r_{n}(\theta) \prod_{1}^{m}\left(1+\lambda_{k} r_{k}(\theta)\right) d \theta \\
& \leqq \max _{m}\left|s_{m}\right| \int_{0}^{1} \prod_{1}^{m}\left(1+\lambda_{k} r_{k}(\theta)\right) d \theta \leqq K .
\end{aligned}
$$

Putting $\lambda_{n}=\operatorname{sign} a_{n}$, we get $\sum\left|a_{n}\right|<\infty$. We proceed now to the general case where

$$
\left|\sum_{1}^{m} a_{n} x_{n}(t)\right| \leqq K .
$$

In the familiar way, we insert the Rademacher functions and we obtain for a fixed $\theta$ 


$$
\left|\sum_{1}^{m} a_{n} x_{n}(t) r_{n}(\theta)\right| \leqq\left|\sum_{1}^{m_{i}} a_{n_{i}} x_{n_{i}}(t)\right|+\sum_{1}^{q_{i}} a_{n_{j}} x_{n_{j}}(t) .
$$

In virtue of Theorem $3,(10)$, and Lemma 2 with $p=\infty$, we have that for each $\theta$ almost everywhere in $t$

$$
\left|\sum_{1}^{m} a_{n} x_{n}(t) r_{n}(\theta)\right| \leqq 2 K .
$$

On account of Fubini's theorem this implies that for almost every $t$

$$
\left|\sum_{1}^{m} a_{n} x_{n}(t) r_{n}(\theta)\right| \leqq C
$$

almost everywhere in $\theta$. In view of the first part of the proof, we obtain almost everywhere in $t$ that $\sum\left|a_{n} x_{n}(t)\right|<\infty$, which completes the proof.

COROLlARY 1. If $\sum_{1}^{m} a_{n} x_{n}(t)$ converges, a.e. to a bounded function, then $\sum\left|a_{n} x_{n}(t)\right|<\infty$ a.e.

This follows immediately from Lemma 2 and the theorem. A further remark in this connection is that if $s_{n}(t) \geqq-A$ almost everywhere, then we assert that $s_{n}(t)$ converges almost everywhere to an integrable function. Indeed

$$
\int_{0}^{1}\left|s_{n}(t)\right| d t \leqq \int_{0}^{1}\left|s_{n}(t)+A\right| d t+\int_{0}^{1}|A| d t=\int_{0}^{1} s_{n}(t) d t+2 A=2 A .
$$

Applying Lemma 2 we obtain our result.

It is of interest to study the relationship of convergence of $\sum_{1}^{m} a_{k} x_{k}(t)$ and $\sum a_{k}^{2}<\infty$.

We say that $\left\{x_{n}(t)\right\}$ possesses the property $C_{p}$ if whenever $\sum_{1}^{m} a_{k} x_{k}(t)$ converges to a function in $L^{p}$, then $\sum a_{k}^{2}<\infty$. It is sufficient to study the property $C_{p}$ only for $1 \leqq p<2$, the case of $p \geqq 2$ being trivial. We now supplement a. result of Marcinkiewicz and Zygmund [4, pp. 65-67].

TheOREM 5. A necessary and sufficient condition that $\left\{x_{n}(t)\right\}$ have property $C_{p}(1 \leqq p<2)$ is that $\lim \inf \int_{0}^{1}\left|x_{n}(t)\right|^{p} d t \geqq \Delta>0$.

Proof. Necessity. Let us suppose the contrary that $\lim \inf \int_{0}^{1}\left|x_{n}(t)\right|^{p} d t=0$, then let $n_{k}$ be determined so that

$$
\int_{0}^{1}\left|x_{n_{k}}(t)\right|^{p} d t \leqq \frac{1}{k^{2}}
$$

Choose $a_{n_{k}}=1$. Otherwise $a_{n}=0$.

Using the inequality that for $x, y, z, \cdots \geqq 0$ and $0<r<1$

$$
(x+y+z+\cdots)^{r}<x^{r}+y^{r}+z^{r}+\cdots,
$$


since $p / 2<1$, we have

$$
\begin{aligned}
\int_{0}^{1}\left|\sum_{1}^{m} a_{n} x_{n}(t)\right|^{p} d t & \leqq A_{p} \int_{0}^{1}\left|\sum a_{n}^{2} x_{n}^{2}(t)\right|^{p / 2} d t \leqq A_{p} \int \sum\left|a_{n} x_{n}(t)\right|^{p} d t \\
& \leqq \int \sum\left|x_{n_{k}}(t)\right|^{p} d t \leqq \sum \frac{1}{k^{2}} \leqq \gamma .
\end{aligned}
$$

In virtue of Lemma 2, we obtain that $\sum_{1}^{m} a_{k} x_{k}(t)$ converges to $f(t) \in L^{p}$. However, since $a_{n}=1$ infinitely often, $\sum a_{n}^{2}<\infty$ which contradicts the hypothesis.

Sufficiency. Since

$$
\left(\int\left(\sum f_{n}\right)^{k}\right)^{1 / k} \geqq \sum\left(\int f_{n}^{k}\right)^{1 / k} \quad \text { for } 0<k<1
$$

where $f_{n} \geqq 0$, we have, since $p / 2<1$, that

$$
\begin{aligned}
\sum_{1}^{m}\left(\int\left|a_{n} x_{n}(t)\right|^{p} d t\right)^{2 / p} & =\sum_{1}^{m}\left(\int\left|a_{n}^{2} x_{n}^{2}(t)\right|^{p / 2} d t\right)^{2 / p} \\
& \leqq\left(\int\left|\sum a_{n}^{2} x_{n}^{2}\right|^{p / 2} d t\right)^{2 / p} \\
& \leqq A_{p}^{\prime}\left(\int_{0}^{1}\left|\sum a_{n} x_{n}(t)\right|^{p}\right)^{2 / p} .
\end{aligned}
$$

If $\sum_{1}^{m} a_{n} x_{n}(t) \rightarrow f(t) \in L^{p}$, then by Lemma 2 and (12), we get

$$
\sum\left|a_{n}\right|^{2}\left(\int\left|x_{n}(t)\right|^{p} d t\right)^{2 / p} \leqq \gamma_{p}
$$

In virtue of the hypothesis, we obtain $\sum a_{n}^{2}<\infty$, which completes the proof.

As a consequence of the proof, we have the following corollary.

Corollary 1. If $\lim \inf \int_{0}^{1}\left|x_{n}(t)\right|^{p} d t \geqq \Delta>0$ for $1 \leqq p<2$, then

$$
\left(\int\left|\sum_{1}^{m} a_{n} x_{n}(t)\right|^{2} d t\right)^{1 / 2} \leqq C_{p}\left(\int\left|\sum_{1}^{m} a_{n} x_{n}(t)\right|^{p} d t\right)^{1 / p}
$$

for any sequence of constants $a_{n}$.

It is well known from the general moment problem that given a system of functions $\left\{\phi_{n}(t)\right\}$, a necessary and sufficient condition that for a sequence of constants $a_{k}$ there exist a function $g(t)$ in $L^{p}$ with $a_{k}=\int g \phi_{k}$ is that

$$
\left|\sum_{1}^{m} a_{k} h_{k}\right| \leqq \gamma\left[\int_{0}^{1}\left|\sum_{1}^{m} h_{k} \phi_{k}(t)\right|^{p^{\prime}} d t\right]^{1 / p^{\prime}}
$$


where $h_{1}, \cdots, h_{m}$ are any numbers and $\gamma$ is an absolute constant. Let us suppose now that $\sum a_{n}^{2}<\infty$; then there exists a function $f(t)$ in $L^{2}$ with $a_{n}$ as Fourier coefficients with respect to $x_{n}(t)$. In virtue of (13) and Corollary 1 , we have that there exists a function in $L^{p^{\prime}}$ having $a_{n}$ as coefficients provided that

$$
\lim \inf \int_{0}^{1}\left|x_{n}(t)\right|^{p} d t \geqq \Delta>0 .
$$

Thus, it follows that:

Corollary 2. If $\lim \inf \int_{0}^{1}\left|x_{n}(t)\right|^{p} d t \geqq \Delta>0$, then if $\sum a_{n}^{2}<\infty$ there exists a function $f(t)$ in $L^{p^{\prime}}(M)$ with $a_{n}$ as its Fourier coefficients.

In the next theorem we shall place an additional restriction on the system, namely:

$$
\lim \inf \int_{0}^{1}\left|x_{n}(t)\right| d t \geqq \Delta>0 .
$$

We have already encountered the same hypothesis in the previous theorem. In essence, the class of systems satisfying (14) includes most important independent systems. Any uniformly bounded orthogonal system has this property. Moreover, as is shown in $\$ 3$, any independent system $x_{n}(t)$ for which there exists a $p>2$ such that

$$
\int_{0}^{1}\left|x_{n}(t)\right|^{p} d t \leqq \gamma \quad(\gamma \text { is an absolute constant })
$$

satisfies (14). Any independent system of functions which are equi-integrable [1] possess this property. In general (14) is assumed in proving most theorems concerning the laws of large numbers and central limit theorems. We have now the following lemma.

LEMma 4. If $\lim$ inf $\int_{0}^{1}\left|x_{n}(t)\right| d t \geqq \Delta>0$, then for any set $E$ of positive measure

$$
\lim \inf \int_{E}\left|x_{n}(t)\right| d t \geqq \Delta>0 .
$$

Proof. If $\sum_{1}^{m} b_{n} x_{n}(t)$ converges in a set of positive measure $E$, it follows from the law of zero and one that it converges almost everywhere. The hypothesis now easily implies that $\sum b_{n}^{2}<\infty$. We have thus shown that $\sum b_{n} x_{n}(t)$ possesses the property that whenever $\sum b_{n} x_{n}(t)$ converges in $E$, then $\sum b_{n}^{2}<\infty$. This gives as in the proof of Theorem 6 that lim inf $\int_{E}\left|x_{n}(t)\right| d t$ $\geqq \Delta>0$.

THEOREM 6. If $\lim \inf \int_{0}^{1}\left|x_{n}(t)\right| d t \geqq \Delta>0, \int_{0}^{1} x_{n}^{2}(t) d t=1$, and if 


$$
\sum\left|a_{n} x_{n}(t)\right|^{p}<\infty
$$

in a set $E$ of positive measure $(1 \leqq p<2), \sum\left|a_{n} x_{n}^{2}(t)\right|^{p}<\infty$ almost everywhere.

Proof. Since $\sum\left|a_{n} x_{n}(t)\right|^{p}<\infty$ in $E$ where $m(E)>0$ there exists a set $E^{\prime} \subset E$ with $m\left(E^{\prime}\right)>0$ such that in $E^{\prime}$

$$
\sum\left|a_{n} x_{n}(t)\right|^{p}<K
$$

In virtue of Lemma 5 , we have

$$
\int_{E^{\prime}}\left|x_{n}(t)\right|^{p} d t \geqq \frac{1}{C} \int_{E}\left|x_{n}(t)\right| d t \geqq \Delta^{\prime}>0 .
$$

Integrating (15) over $E^{\prime}$ with use of (16) yields $\sum\left|a_{n}\right|^{p}<\infty$. Integrating, we obtain

$$
\int_{0}^{1}\left|\sum a_{n} x_{n}(t)\right|^{p} d t \leqq \sum\left|a_{n}\right|^{p} \int x_{n}^{2}(t) d t<\infty .
$$

This easily yields that $\sum\left|a_{n} x_{n}(t)\right|^{p}$ converges almost everywhere.

Corollary 1. If $\int x_{n}^{2}(t) d t=1, \lim \inf \int_{0}^{1}\left|x_{n}(t)\right| d t \geqq \Delta>0$, then the absolute convergence of the series $\sum a_{n} x_{n}(t)$ in a set of positive measure implies the absolute convergence almost everywhere.

This is precisely the theorem for the case $p=1$.

2. Summability. One of the fundamental concepts essential in studying orthogonal systems is the Lebesgue kernel $L_{n}(t)$

$$
L_{n}(t)=\int_{0}^{1}\left|\sum_{1}^{n} \phi_{k}(\theta) \phi_{k}(t)\right| d \theta .
$$

It is customary to assume in working with an orthogonal set of functions that the Lebesgue kernel is uniformly summable by some finite row Toeplitz matrix $[6$, p. 350], that is,

$$
\int_{0}^{1}\left|\sum_{k=1}^{n_{i}} b_{i k} \sum_{l=1}^{k} \phi_{l}(\theta) \phi_{l}(t)\right| d \theta \leqq M \quad \text { a.e. }
$$

In this connection we turn to investigate the character of the Lebesgue kernel of an independent system of functions. We assume first that $\int_{0}^{1} x_{n}(t) d t$ $=0$ for each $n$. We develop many results prior to this investigation.

LEMMA a. If $\sigma_{i}=\sum_{k=1}^{n_{i}} b_{i k} \sum_{l=1}^{k} \epsilon_{l} a_{l}$ converges for every $\epsilon_{l}= \pm 1$, then $\sum\left|a_{l}\right|$ $<\infty$.

Proof. We first establish that if $\sum_{k=1}^{n_{i}} A_{i k} \epsilon_{k}=A_{i}$ converges for every variation of sign $\epsilon_{l}= \pm 1, \sum_{k=1}^{\infty}\left|A_{i k}\right| \leqq C$ for every $i$. If we observe that $\left\{\left(A_{i k}\right)_{k}\right.$ $\left.=x_{i}\right\}_{i}$ is a sequence of elements in $(l)$ (space of absolutely convergent series), 
then the hypothesis implies that $x_{i}$ converges weakly for all functionals $f$ of the form $f=\left\{\epsilon_{k}\right\}$. A result of Banach [11, pp. 138-139] implies that $x_{i}$ are strongly convergent and hence $\left\|x_{i}\right\|=\sum_{k=1}^{n_{i}}\left|A_{i k}\right| \leqq C$. We now complete the proof. Since

$$
\sum_{k=1}^{n_{i}} b_{i k} \sum_{l=1}^{k} \epsilon_{l} a_{l}=\sum_{l=1}^{n_{i}} \epsilon_{l}\left[a_{l} \sum_{k=l}^{n_{i}} b_{i k}\right]=\sum_{l=1}^{n_{i}} \epsilon_{l} A_{i l}
$$

converges for every $\epsilon_{l}= \pm 1$, we have in view of the preceding, that

$$
\left|\sum_{k=1}^{n_{i}} b_{i k} \sum_{l=1}^{k} \epsilon_{l} a_{l}\right| \leqq \sum_{l=1}^{n_{i}}\left|a_{l} \sum_{k=1}^{n_{i}} b_{i k}\right|=\sum_{l=1}^{n_{i}}\left|A_{i l}\right| \leqq C .
$$

If we replace $\epsilon_{l}$ by $r_{l}(\theta)$, then the hypothesis states that $\sigma_{i}(\theta)$ converges for every $\theta$. In virtue of a known result $[10, \mathrm{pp} .122-125]$, this implies that $s_{m}(\theta)=\sum_{l=1}^{m} a_{l} r_{l}(\theta)$ converges a.e. This, combined with (a), yields on account of the proof of Theorem 4 that $\sum\left|a_{n}\right|<\infty$. This completes the proof.

LEMmA 5. If $\sigma_{i}(t)=\sum_{k=1}^{n_{i}} b_{i k} \sum_{l=1}^{k} a_{l} x_{l}(t)$ and $\lim _{i, j \rightarrow \infty} \int_{0}^{1}\left|\sigma_{i}(t)-\sigma_{j}(t)\right|^{p} d t=0$ for $p \geqq 1$, then

$$
\int_{0}^{1}\left|s_{n}(t)\right|^{p} d t \leqq \gamma
$$

Proof. If $\sigma_{i}^{\prime}(t)=\sum_{k=1}^{n_{i}} b_{i k} \sum_{l=1}^{k} \epsilon_{l} a_{l} x_{l}(t)$ with $\epsilon_{l}= \pm 1$ then a double application of Lemma 1 and the hypothesis of the lemma yields that

$$
\lim _{i, j \rightarrow \infty} \int_{0}^{1}\left|\sigma_{i}^{\prime}(t)-\sigma_{j}^{\prime}(t)\right|^{p} d t=0
$$

for any $\epsilon_{l}= \pm 1$. This implies that for any $b_{k}$ the Fourier coefficients of any functions in $L^{p^{\prime}}(M)$, we have that

$$
\lim _{i} \sum_{k=1}^{n_{i}} b_{i k} \sum_{l=1}^{k} \epsilon_{l} a_{l} b_{l}
$$

exists. In virtue of Lemma a, this gives that $\int_{0}^{1}\left|s_{n}(t)\right|^{p} d t \leqq \gamma$, for otherwise, there exists a function in $L^{p^{\prime}}(M)$ such that $\left|\sum a_{k} b_{k}\right|=\infty$, which is impossible.

It is clear in virtue of Lemma 5 and Lemma 2 that:

LEMмA 6. If $\int\left|\sigma_{i}(t)-\sigma_{j}(t)\right|^{p} d t \rightarrow 0$ for $p \geqq 0$, then

$$
\int\left|s_{i}(t)-s_{j}(t)\right|^{p} \rightarrow 0 \text {. }
$$

Furthermore, we establish the following lemma.

LEMma 7. If $\int_{0}^{1}\left|\sigma_{i}(t)\right|^{p} d t \leqq \gamma$ for $p>1$, then 


$$
\int_{0}^{1}\left|s_{n}(t)\right|^{p} d t \leqq \gamma^{\prime} \quad \text { for } p>1 .
$$

Proof. The hypothesis implies the existence of a subsequence $\sigma_{i_{n}}(t)$ which converges weakly in $L^{p}$. The selection of a subsequence can be considered as a Toeplitz matrix $B$ operating on $\sigma_{i}(t)$. If $A$ denotes the original Toeplitz matrix then $B A\left\{s_{n}(t)\right\}=\sigma_{i_{n}}(t)$, where $B A=C$ is a new Toeplitz matrix. Thus, we have shown the existence of a Toeplitz matrix whose elements $\sigma_{i}^{\prime}(t)=C\left\{s_{n}(t)\right\}$ converge weakly in $L^{p}(p>1)$. Due to a result of Saks [9, p. 51], we can select a subsequence from $\sigma_{i}^{\prime}(t)$ which is strongly $(C, 1)$ summable in $L^{p}$, that is,

$$
\int_{0}^{1}\left|\frac{\sum_{1}^{m} \sigma_{i_{k}}^{\prime}(t)}{m}-\frac{\sum_{k=1}^{n} \sigma_{i_{k}}^{\prime}(t)}{n}\right|^{p} \rightarrow 0 \quad \text { as } m, n \rightarrow \infty .
$$

Again, as above, this reduces to a new Toeplitz matrix $E$ with the properties

$$
E\left\{s_{n}(t)\right\}=\sigma_{i}^{\prime \prime}(t) \quad \text { such that } \quad \int\left|\sigma_{i}^{\prime \prime}-\sigma_{j}^{\prime \prime}\right|^{p} \rightarrow 0 .
$$

In view of Lemma 5, we obtain the result.

The proof of this lemma is not applicable to $p=1$.

We now use a familiar method introduced by Marcinkiewicz and Zygmund $[5$, p. 111].

LEMMA 8. If for any Toeplitz matrix, $\sigma_{n}$ converges almost everywhere to $f(t)$ which is a function in $L^{p}(p \geqq 1)$, then $s_{n}$ converges.

Proof. We introduce an independent system $x_{1}(t), x_{1}^{\prime}(t), \cdots, x_{n}(t)$, $x_{n}^{\prime}(t), \cdots$ with $x_{n}^{\prime}(t)$ equimeasurable to $-x_{n}(t)$ respectively $[4$, p. 63$]$. We shall consider the symmetric independent system $\overline{x_{n}(t)}=x_{n}+x_{n}^{\prime}$. In view of the hypothesis, $\sum_{1}^{k} a_{n} \bar{x}_{n}(t) r_{n}(\theta)$ is summable for each $\theta$ to a $p$ th integrable function $f(t, \theta)$. Moreover, it is clear from the properties of $\bar{x}_{n}(t)$ that for any $\theta_{1}$ and $\theta_{2}$

$$
\int_{0}^{1}\left|f\left(t, \theta_{1}\right)\right|^{p} d t=\int_{0}^{1}\left|f\left(t, \theta_{2}\right)\right|^{p} d t
$$

Hence, if we average over $\theta$, we obtain

$$
\int_{0}^{1} d \theta \int_{0}^{1}|f(t, \theta)|^{p} d t \leqq \gamma
$$

In virtue of the Fubini theorem, we have that for almost every $t$, $\sum_{1}^{k} a_{n} \bar{x}_{n}(t) r_{n}(\theta)$ is almost everywhere summable to $f(t, \theta)$ in $\theta$. In virtue of a well known result $[10$, p. 122$]$, we obtain that $\sum_{1}^{k} a_{n} \bar{x}_{n}(t) r_{n}(\theta)$ converges almost everywhere in $\theta$ for almost every $t$ to $f(t, \theta)$ in $L^{p}$. Moreover, in view of 
Lemma 2, we obtain

$$
\int_{0}^{1}\left|\sum_{1}^{k} a_{n} \bar{x}_{n}(t) r_{n}(\theta)\right|^{p} d \theta \leqq A \int_{0}^{1}|f(t, \theta)|^{p} d \theta .
$$

On account of (17), if we integrate we get

$$
\int_{0}^{1} d t \int_{0}^{1}\left|\sum_{1}^{k} a_{n} \bar{x}_{n}(t) r_{n}(\theta)\right|^{p} d \theta \leqq \gamma^{\prime}
$$

Using Lemma 1 twice, we have that

$$
\int_{0}^{1}\left|\sum_{1}^{k} a_{n} \bar{x}_{n}(t)\right|^{p} d t \leqq C
$$

which in virtue of a known result $[5$, p. 110] implies

$$
\int_{0}^{1}\left|\sum_{1}^{k} a_{n} x_{n}(t)\right|^{p} d t \leqq C .
$$

Finally, applying Lemma 2 gives the result.

LEMMA 9. If $\sigma_{i}(t)$ is asymptotically convergent to a function in $L^{p}$, then $s_{n}(t)$ converges.

Proof. This follows easily from Lemma 8 since the hypothesis insures the existence of a subsequence $\sigma_{i_{k}}$ convergent to a function in $L^{p}$. This can be represented as a new Toeplitz matrix operating on $s_{n}(t)$ whose elements converge to a function in $L^{p}$. It remains only to apply the preceding lemma.

Lemmas 6, 7, 8 and 9 yield the following theorem.

TheOREM 7. If for any Toeplitz matrix either

(a) $\sigma_{i}(t)$ converges strongly in $L^{p}, p \geqq 1$,

(b) $\sigma_{i}(t)$ converges weakly in $L^{p}, p>1$,

(c) $\sigma_{i}(t)$ converges a.e. to a function in $L^{p}, p \geqq 1$, or

(d) $\sigma_{i}(t)$ converges asymptotically to a function in $L^{p}(p \geqq 1)$, then $s_{n}(t)$ converges to a function in $L^{p}$.

We now present two corollaries to Theorem 7.

COROLlARY 1. If $\left|\sum_{k=1}^{m_{i}} b_{i k} \sum_{l=1}^{k} a_{l} x_{l}(t)\right| \leqq M$ almost everywhere, then a.e.

$$
\sum\left|a_{l} x_{l}(t)\right|<\infty \text {. }
$$

Proof. The hypothesis implies in virtue of Theorem 7 that

$$
\left|\sum_{1}^{m} a_{l} x_{l}(t)\right| \leqq M
$$


almost everywhere. An application of Theorem 4 gives the result.

COROLlary 2. If for $p \geqq 1$

$$
\int_{0}^{1}\left|\sum_{k=1}^{n_{i}} b_{i k} \sum_{n=1}^{k} x_{n}(t) x_{n}(\theta)\right|^{p} d t \leqq M
$$

almost everywhere, then

$$
\int_{0}^{1}\left|\sum_{1}^{k} x_{n}(t) x_{n}(\theta)\right|^{p} d t \leqq M
$$

a.e., where $b_{i k}$ is a finite row matrix.

Proof. It follows easily on account of the hypothesis that a.e.

$$
\left|\sum_{k=1}^{n_{i}} b_{i k} \sum_{n=1}^{k} b_{n} x_{n}(t)\right| \leqq M
$$

for every $b_{n}$ which are the Fourier coefficients of a function in $L^{p^{\prime}}$. Moreover, (a) yields that

$$
\int_{0}^{1}\left|\sum_{k=1}^{n_{i}} b_{i k} \sum_{n=1}^{k} b_{n} x_{n}(t)\right|^{p} d t \leqq \gamma
$$

This implies in virtue of Theorem 10 that $s_{n}(t)$ converges almost everywhere. Consequently, in view of (a), we get a.e.

$$
\left|\sum_{n=1}^{k} b_{n} x_{n}(t)\right| \leqq K
$$

The conclusion of the corollary follows easily from this fact.

We remark now that in view of Lemma 8 all the preceding theorems of this chapter are valid for infinite Toeplitz matrices.

We indicate now how (b) in Theorem 10 can be extended to the case $p=1$. We do this for a finite row positive Toeplitz matrix. First, we specialize even further to the case where $\sum_{k=1}^{n_{i}} b_{i k}=1$ for all $i$. The properties of a Toeplitz matrix insure the existence of a subsequence of rows for which

$$
\sum_{k=l}^{n_{m_{i}}} b_{m_{i} k} \leqq \sum_{k=l}^{n_{m_{i+1}}} b_{m_{i+1}, k} \quad \text { for every } l .
$$

For convenience of notation, we shall assume that all rows of the Toeplitz matrix have this property. In virtue of the hypothesis (b) and Lemma 1, we obtain

$$
\int_{0}^{1}\left|\sum_{1}^{n_{i}}\left(a_{l} x_{l}(t)\right)^{2}\left(\sum_{k=l}^{n_{i}} b_{i k}\right)^{2}\right|^{1 / 2} d t \leqq \gamma .
$$

If $F_{i}=\sum_{l=1}^{n_{i}}\left(a_{l} x_{l}(t)\right)^{2}\left(\sum_{k=l}^{n_{i}} b_{i k}\right)^{2}$ then from (18) it follows that 


$$
F_{i} \leqq F_{i+1} \leqq F_{i+2} \leqq \cdots \quad \text { with } F_{i} \in L^{1 / 2}
$$

Interchanging limit with integral, we obtain

$$
\int_{0}^{1}\left|\sum_{1}^{m}\left(a_{l} x_{l}(t)\right)^{2}\right|^{1 / 2} d t \leqq \gamma .
$$

Again by Lemma 1, we have

$$
\int\left|\sum_{1}^{n} a_{l} x_{l}(t)\right| d t \leqq \gamma^{\prime}
$$

whence on using Lemma 2 , the result follows.

The case where $\sum_{k=1}^{n_{i}} b_{i k} \neq 1$ but $b_{i k} \geqq 0$ can be easily reduced to a similar circumstance so as to yield (22).

TheOREM 8. If $\lim \inf \int_{0}^{1}\left|x_{n}(t)\right| d t \geqq \Delta>0$ and $\sigma_{i}(t)$ converges in a set of positive measure, then $s_{n}(t)$ converges almost everywhere.

Proof. Using the procedure of Lemma 8 , we obtain that $\sum_{1}^{k} a_{k} \bar{x}_{k}(t) r_{k}(\theta)$ is summable for each $t$ of a set of positive measure in a set of positive measure of $\theta$. This gives by a known result $[10, \mathrm{p} .122]$ that $\sum a_{n}^{2} \bar{x}_{n}^{2}(t)<\infty$ for $t$ in a set of positive measure $E$. Consequently, there exists a set of positive measure $E^{\prime}$ for which

$$
\sum a_{n}^{2} \bar{x}_{n}^{2}(t) \leqq k
$$

Since

$$
\int_{0}^{1}\left|\bar{x}_{n}(t)\right| d t \geqq A_{p} \int_{0}^{1}\left|x_{n}(t)\right| d t \geqq \Delta>0
$$

$[5$, p. 110$]$, we have, Lemma 5 applied to $\bar{x}_{n}(t)$, after integrating that $\sum a_{n}^{2}$ $<\infty$. It follows immediately from this that $\sum_{1}^{m} a_{n} x_{n}(t)$ converges a.e.

We close this chapter with the discussion of the Lebesgue kernel of an independent system.

ThEOREM 9. If $\lim$ inf $\int_{0}^{1}\left|x_{n}(t)\right| d t \geqq \Delta>0$, then there exists no finite row Toeplitz matrix which sums the kernel of an independent system. That is to say it is impossible that a.e. we have

$$
\int_{0}^{1}\left|\sum_{k=1}^{n_{i}} b_{i k} \sum_{l=1}^{k} x_{l}(t) x_{l}(\theta)\right| d t \leqq M .
$$

Proof by contradiction. In virtue of Corollary 2 to Theorem 7, we infer that a.e.

$$
\int_{0}^{1}\left|\sum_{1}^{k} x_{l}(t) x_{l}(\theta)\right| d t \leqq M .
$$


In view of Lemma 1, we obtain that a.e.

$$
\int_{0}^{1}\left|\sum x_{l}^{2}(t) x_{l}^{2}(\theta)\right|^{1 / 2} d t \leqq C
$$

or for almost every $\theta$ in $E$ with $m(E)=1$

$$
\sum x_{l}^{2}(\theta) x_{l}^{2}(t)
$$

converges almost everywhere in $t$. Applying, for $\theta \in E$, the Egoroff Theorem and Lemma 5, we secure that $\sum x_{l}^{2}(\theta)$ converges a.e. Another application of the Egoroff Theorem and Lemma 5 gives that $\sum_{1}^{\infty} 1<\infty$, which is impossible.

In the case when $x_{n}(t)$ are uniformly bounded, it can be shown that there can exist no Toeplitz matrix such that

$$
\int\left|\sum_{k=1}^{n_{i}} b_{i k} \sum_{l=1}^{k} x_{l}(\theta) x_{l}(t)\right| d t \leqq M
$$

in a set $E$ of $\theta$ of positive measure.

This result draws a sharp contrast between the kernel of an independent system and the kernel of the Walsh system (completion of Rademacher series) whose kernel is summable by the Cesàro matrix.

3. Independence and lacunarity. We now exhibit several connections of lacunary orthogonal systems with independent systems. We first proceed to define what constitutes a lacunary orthogonal system.

We assume throughout this chapter that

$$
\int_{0}^{1} x_{n}(t) d t=0, \quad \int_{0}^{1} x_{n}^{2}(t) d t=1 .
$$

An orthogonal system $\phi_{n}(t)$ is said to be lacunary of order $p>2$ if whenever $\sum a_{n}^{2}<\infty$, then $\sum a_{n} \phi_{n}(t)$ converges strongly in $L^{p}$. It has been shown by Steinhaus that this is equivalent to

$$
\left(\int\left|\sum_{1}^{m} a_{n} x_{n}(t)\right|^{p} d t\right)^{1 / p} \leqq \mu_{p}\left(\sum a_{n}^{2}\right)^{1 / 2}
$$

[6, chap. 7]. We show first:

ThEOREM 10. A necessary and sufficient condition that an independent system $x_{n}(t)$ be lacunary of order $p(p>2)$ is that $\int_{0}^{1}\left|x_{n}(t)\right|^{p} d t \leqq \gamma$ for all $n$.

Proof. Sufficiency. Using the Minkowski inequality

$$
\left(\int\left(\sum f_{n}\right)^{p}\right)^{1 / p} \leqq \sum\left(\int f_{n}^{p}\right)^{1 / p} \quad \text { for } p>1 \text { and } f_{n} \geqq 0,
$$

we have in virtue of Lemma 1 , since $p / 2>1$, 


$$
\begin{aligned}
\left(\int_{0}^{1}\left|\sum a_{n} x_{n}(t)\right|^{p} d t\right)^{2 / p} & \leqq A_{p}\left(\int\left|\sum a_{n}^{2} x_{n}^{2}(t)\right|^{p / 2} d t\right)^{2 / p} \\
& \leqq A_{p} \sum\left(\int\left(a_{n}^{2} x_{n}^{2}(t)\right)^{p / 2}\right)^{2 / p} \\
& \leqq A_{p} \sum a_{n}^{2}\left(\int_{0}^{1}\left|x_{n}(t)\right|^{p} d t\right)^{2 / p} \leqq \gamma_{p} \sum a_{n}^{2}
\end{aligned}
$$

Thus (19) has been established, which completes the proof of sufficiency.

Necessity. If $f \in L^{p^{\prime}}(p>2)$ and $a_{n}=\int_{0}^{1} f x_{n}(t) d t$, then

$$
\sum_{1}^{m} a_{n}^{2}=\int_{0}^{1} f \sum_{1}^{m} a_{n} x_{n}(t) d t \leqq\left(\int_{0}^{1}|f|^{p^{\prime}}\right)^{1 / p^{\prime}}\left(\int\left|\sum_{1}^{m} a_{n} x_{n}(t)\right|^{p} d t\right)^{1 / p} .
$$

This implies with the aid of (19) that

$$
\sum_{1}^{m} a_{n}^{2} \leqq\left(\int|f|^{p^{\prime}}\right)^{1 / p^{\prime}} \mu_{p}\left(\sum_{1}^{m} a_{n}^{2}\right)^{1 / 2}
$$

or

$$
\left(\sum_{1}^{m} a_{n}^{2}\right)^{1 / 2} \leqq \mu_{p}\left(\int|f|^{p^{\prime}}\right)^{1 / p^{\prime}}
$$

Consequently, $\sum a_{n}^{2}<\infty$, hence $\left|a_{n}\right| \leqq M$. Suppose $\lim \sup \int_{1}^{0}\left|x_{n}(t)\right|^{p} d t=\infty$, then there exists a function $f \in L^{p \prime}$ such that lim sup $\left|\int_{1}^{0} f x_{n}(t) d t\right|=\infty$, which is a contradiction. Q.E.D.

In particular if $x_{n}(t)$ is uniformly bounded the system is lacunary of every order greater than 2. This also follows from Lemma 1. Moreover, the proof of the sufficiency establishes the following corollary.

CoROllaRY 1. If $\int_{0}^{1}\left|x_{n}(t)\right|^{p} d t \leqq \gamma$ for $p>2$, then

$$
\left(\int_{0}^{1}\left|\sum_{1}^{m} a_{n} x_{n}(t)\right|^{p} d t\right)^{1 / p} \leqq \gamma_{p}\left(\int\left|\sum_{1}^{m} a_{n} x_{n}(t)\right|^{2}\right)^{1 / 2} \text {. }
$$

The hypothesis $\int_{0}^{1}\left|x_{n}(t)\right|^{p} d t \leqq \gamma$ if applied to any orthogonal system guarantees the existence of a subsequence $x_{n_{k}}$ which forms a lacunary system [6, chap. 7].

CoRollary 2. If for every function $f \in L^{p^{\prime}}\left(1<p^{\prime}<2\right)$ with $a_{n}=\int f x_{n}$ we have $\left|a_{n}\right| \leqq M_{f}$, then $\sum a_{n}^{2}<\infty$ for every function in $L^{p^{\prime}}$.

Proof. The hypothesis clearly implies $\left(\int\left|x_{n}(t)\right|^{p} d t\right)^{1 / p} \leqq \gamma$, whence as in the proof we obtain $\sum a_{n}^{2}<\infty$.

CoRollary 3. If $x_{n}(t)$ is lacunary of order $p>2$, and if $\sum\left|a_{n} x_{n}(t)\right|^{a}<\infty$ 
for a set $E$ of positive measure, then $\sum\left|a_{n} x_{n}(t)\right|^{a}<\infty$ almost everywhere.

Proof. Since $x_{n}(t)$ is lacunary, it follows that $\int_{0}^{1}\left|x_{n}(t)\right| \geqq \Delta>0$ [6, chap. 7].

The remainder of the proof follows as in Theorem 6 .

We present other conditions for lacunarity.

THEOREM 11. If $\int_{0}^{1}\left|x_{n}(t)\right| p^{\prime} d t \geqq \Delta>0(p>2)$, then a necessary and sufficient condition that $x_{n}(t)$ is lacunary of order $p$ is that the expansion of every $f \in L^{p}$ converge to a function in $L^{p}$.

REMARK. Since $\sum a_{n}^{2}<\infty$, the expansion of every $f \in L^{p}$ necessarily converges.

Necessity. Since $x_{n}(t)$ is lacunary, we have for any $a_{n}$ the Fourier coefficient of $f \in L^{p}$

$$
\int_{0}^{1}\left|\sum_{1}^{m} a_{n} x_{n}(t)\right|^{p} d t \leqq k_{p}\left(\sum a_{n}^{2}\right)^{p / 2} \leqq \gamma .
$$

An application of Lemma 2 gives the result.

Sufficiency. In virtue of Lemma 2, we have for every $f \in L^{p}$

$$
\int_{0}^{1}\left|s_{n}(t)\right|^{p} d_{i} \leqq \gamma
$$

In view of Theorem 1 , we have for every $f$ in $L^{p}$

$$
\int_{0}^{1}\left|s_{n}(f)-s_{m}(f)\right|^{p} d t \rightarrow 0
$$

This gives for every $g(t)$ in $L^{p^{\prime}}$

$$
\int_{0}^{1}\left|s_{n}(g)-s_{m}(g)\right| p^{\prime} d t \rightarrow 0
$$

Whence, we have

$$
\left|b_{n}\right|\left(\int_{0}^{1}\left|x_{n}(t)\right|^{p^{\prime}}\right)^{1 / p^{\prime}} \leqq k
$$

or

$$
\left|b_{n}\right| \leqq k_{f}
$$

As a consequence of Corollary 2 to Theorem 12 the lacunarity of order $p$ follows easily from this fact.

It is well known that lacunary trigonometric series behave like independent series and we expect most of the results of the three chapters to remain valid for such series. It is first necessary to remark that for general Fourier series, we have the inequality 


$$
\int_{0}^{1} \max _{k}\left|s_{n_{k}}(t)\right|^{p} d t \leqq k_{p} \int|f|^{p} \quad \text { for } p>1
$$

where

$$
\begin{gathered}
s_{n_{k}}=\frac{a_{0}}{2}+\sum_{1}^{n_{k}}\left[a_{n} \cos n x+b_{n} \sin n x\right], \\
n_{k+1} \geqq \lambda n_{k} \text { for } \lambda>1, \quad b_{n}=\int f \frac{\cos n x}{\sin n x} d x .
\end{gathered}
$$

In the case of lacunary trigonometric series the ordinary partial sums of such series coincide with $s_{n_{k}}$. Moreover, for lacunary trigonometric series, we have

$$
A_{p}\left(\sum a_{n}^{2}+b_{n}^{2}\right)^{p / 2} \leqq \int\left|s_{n}\right|^{p} \leqq A_{p}^{\prime}\left(\sum a_{n}^{2}+b_{n}^{2}\right)^{p / 2}
$$

Clearly, (20) and (21) occupy the analogous role for the inequalities of Lemma 1 . In the case of Lemma 5 , it suffices to remark that if a lacunary trigonometric series is summable by a Toeplitz matrix in a set of positive measure it converges almost everywhere.

Thus the methods of this paper yield many new proofs of known theorems concerning lacunary trigonometric series.

For a general lacunary orthogonal system it is now known whether

$$
\int_{0}^{1} \max _{1 \leqq n \leqq m}\left|s_{n}(t)\right|^{p} d t \leqq k_{p} \int_{0}^{1}\left|s_{m}(t)\right|^{p} d t
$$

holds and hence we cannot assert the validity of the results of this paper for such systems of functions.

\section{REFERENCES}

1. A. Marcinkiewicz, Indépendantes fonctions I, II and III, Fund. Math. (1938, 1939).

2. H. Steinhaus, Sur la probabilité de la convergence de series, Studia Math. vol. 2 (1930).

3. M. Kac, Sur les fonctions indépendantes I, Studia Math. vol. 6 (1936). (1937).

4. J. Marcinkiewicz and A. Zygmund, Sur les fonctions indépendantes, Fund. Math. vol. 29

5. - Quelques theoremes sur les fonctions independantes, Studia Math. vol. 7 (1938).

6. S. Kaczmarz and H. Steinhaus, Theorie der Orthogonalreihen, Warsaw, 1935.

7. M. Kac and H. Steinhaus, Sur les fonctions independantes II, Studia Math. vol. 6 (1936).

8. W. Orlicz, Über Folgen linearer Operationen, die von einem Parameter abhangen, Studia Math. vol. 5 (1934). (1930)

9. S. Saks and S. Banach, Sur la convergence forte dans les champs, Studia Math. vol. 2

10. A. Zygmund, Trigonometrical series, 1935.

11. S. Banach, Theorie des linear operations, Warsaw, 1932.

Princeton University,

Princeton, N. J. 